\title{
A randomized, single-blind cross-over design evaluating the effectiveness of an individually defined, targeted physical therapy approach in treatment of children with cerebral palsy
}

Clinical Rehabilitation 2014, Vol. 28(I0) 1039-1052 (C) The Author(s) 2014 Reprints and permissions: sagepub.co.uk/journalsPermissions.nav DOI: $10.1177 / 0269215514544984$ cre.sagepub.com

@SAGE

\author{
Inge Franki' ${ }^{1,2}$, Christine Van den Broeck', \\ Josse De Cat ${ }^{2,3,4}$, Wieke Tijhuis ${ }^{2}$, Guy Molenaers ${ }^{2,3,4}$, \\ Guy Vanderstraeten' and Kaat Desloovere ${ }^{2,3}$
}

\begin{abstract}
Objective: A pilot study to compare the effectiveness of an individual therapy program with the effects of a general physical therapy program.

Design: A randomized, single-blind cross-over design.

Participants: Ten ambulant children with bilateral spastic cerebral palsy, age four to nine years.

Intervention: Participants were randomly assigned into a ten-week individually defined, targeted or a general program, followed by a cross-over.

Main outcome measures: Evaluation was performed using the Gross Motor Function Measure- 88 and three-dimensional gait analysis. General outcome parameters were Gross Motor Function Measure-88 scores, time and distance parameters, gait profile score and movement analysis profiles. Individual goal achievement was evaluated using z-scores for gait parameters and Goal Attainment Scale for gross motor function.

Results: No significant changes were observed regarding gross motor function. Only after individualized therapy, step- and stride-length increased significantly $(p=0.022 ; p=0.017)$. Change in step-length was higher after the individualized program $(p=0.045)$. Within-group effects were found for the pelvis in transversal plane after the individualized program $(p=0.047)$ and in coronal plane after the general program $(p=0.047)$. Between-program differences were found for changes in the knee in sagittal plane, in the advantage of the individual program $(p=0.047)$. A median difference in z-score of 0.279 and 0.419 was measured after the general and individualized program, respectively. Functional goal attainment was higher after the individual therapy program compared with the general program (48 to 43.5).

Conclusion: The results indicate slightly favorable effects towards the individualized program. To detect clinically significant changes, future studies require a minimal sample size of 72 to 90 participants.
\end{abstract}

'Rehabilitation Sciences and Physiotherapy, Ghent University, Ghent, Belgium

${ }^{2}$ Department of Rehabilitation Sciences, K.U.Leuven, Belgium

${ }^{3}$ Clinical Motion Analysis Laboratory, University Hospital

Leuven, Pellenberg, Belgium
${ }^{4}$ Department of Pediatric Orthopaedics, K.U.Leuven, Belgium

Corresponding author:

Inge Franki, Rehabilitation Sciences and Physiotherapy Gent, Campus Heymans (UZ) IB3, De Pintelaan 185, Gent 9000,

Belgium.

Email: inge.franki@ugent.be 


\section{Keywords}

Cerebral palsy, physical therapy, goal-setting; individualized therapy

Received: 10 February 20I4; accepted: 3 July 2014

\section{Introduction}

Many different techniques and approaches are available for physical therapy treatment of children with cerebral palsy. ${ }^{1}$ Although promising results are available for isolated physical therapy techniques, evidence about the outcome of complete programs using a combination of different techniques is more limited. ${ }^{2,3}$

Several research studies have evaluated the effects of goal-setting in physical therapy of children with cerebral palsy. ${ }^{4-9}$ The results of a recent systematic review, however, could not provide support for the effectiveness of goal-setting within an activity-focused approach on treatment outcome. ${ }^{10}$ The authors of this review found that none of the included studies were designed specifically to determine the effect of goal-setting per se, as goalsetting was additional to guiding the interventions.

An important advantage of goal-oriented physical therapy however, is the potential benefit to enhance training specificity. Goal planning, in that context, can be used to identify the tasks and contexts of particular interest and thereby, to use a more targeted and focused approach. ${ }^{11}$

In a previous study, we developed and validated a clinical decision framework based on the hypothesisoriented algorithm for clinicians ${ }^{12}$ and the International Classification of Functioning, Disability and Health (ICF) for Children and Youth, ${ }^{13,14}$ Organizing assessment results within the framework of the International Classification of Functioning provides a profound and complete overview of the child and results in an objective problem list for the child. In children with cerebral palsy, however, this list is generally very extensive. Therefore, as a therapist, one has to prioritize and select possible and realistic targets in treatment. The hypothesis-oriented algorithm for clinicians provided this strategy-oriented structure for clinical reasoning and was used to reduce the long problem list to a selection of main problems. The combined approach of both frameworks was demonstrated as a positive structure that supported clinical reasoning in pediatric physical therapists. ${ }^{14}$

As a next step, a pilot study was set up to use this clinical reasoning structure in a controlled intervention. This study, therefore, aims to investigate the effectiveness of an individually defined and targeted physical therapy approach using structured clinical reasoning on gait and gross motor function of ambulant children with bilateral spastic cerebral palsy. Using a randomized, repeatedmeasures, cross-over design, the outcome of this approach is compared with the outcome of a general program.

It is hypothesized that an individually defined targeted approach, based on structured clinical reasoning and including the results of threedimensional gait analysis, provides better results than a general program.

\section{Method}

Participants were selected through the cerebral palsy reference center of the University Hospital of Leuven. Inclusion criteria were diagnosis of bilateral spastic cerebral palsy, Gross Motor Function Classification System Level I, II or III, age between four and nine years and sufficient cooperation to understand and execute simple verbal instructions. Patients were excluded when they showed severe muscle contractures or bony deformities, a history of multilevel orthopedic surgery or additional disorders that would make participation to the physical therapy programs not feasible (e.g. severe cognitive disorders, deafness, blindness, etc.). The programs were planned at a time when children had not received botulinum-toxin A injections within the previous six months. A convenience 
sample of 10 children represented a manageable number to reassure an adequate follow-up for this pilot study. Children were recruited between January 2010 and January 2012.

The programs were executed by the child's personal physical therapist, who agreed to precisely follow the prescribed program. A physical therapy degree was required, no further specialization was mandatory. The therapist and parent or caretaker of the child signed an informed consent. The study was approved by the ethical committee of the University Hospital of Leuven.

At the start of the study, the selected group of 10 children was followed for a period of 10 weeks of usual care. During this period, usual physical therapy of the child was continued without a specific intervention. A diary was provided to document the content of the therapy sessions. The therapists were asked to fill in the diary after each session, to register how much of the therapy time targeted the impairment, activity and participation level.

Subsequently, the children were enrolled in the actual intervention study. For this part of the study, an alternating treatment design with randomization and cross-over was set up. A first treatment program, general or individualized, was presented to the participants based on an independent randomization procedure. Randomization was performed by a person blinded and independent to the study, and was based on the principles of minimization, as described by Altman and Bland. ${ }^{15}$ After completing one program, a wash-out period of minimally 10 weeks usual care was continued. Consequently, each child participated in the remaining program. The children that originally received the individualized program, now received the general program and vice versa (Figure 1).

The general treatment program was based on the most common aims in children with bilateral spastic cerebral palsy of the involved age-range ${ }^{16}$ and was the same for all children. The main aims were in accordance with the clinical management recommendations for children with spastic diplegia as stated by the task force of the American Physical Therapy Association. ${ }^{16}$ The general considerations reported in this statement, are musculoskeletal and neuromuscular systems including strength, range of motion, posture, balance, motor function, muscle tone and movement patterns. The program, therefore, contained exercises to improve strength, selectivity and mobility and included a set of functional exercises to encourage mobility and play.

The individually defined, targeted programs (further referred to as "individualized programs") were designed to target the specific main problems of the child, tailored to the needs of the child and specific problems at the different levels of the ICF at that particular moment. Definition of the individual problems was based on the results of all assessments. At the level of body structure and function, results of the clinical examination were considered. At activity level, three-dimensional gait analysis and gross motor function evaluation was used, complemented with a structured interview with the parents and child. The results of the gait analysis thereby played a major role in unraveling the motor problems of the child and the identification of the main problems. The programs were based on the clinical decision framework as described by Franki et al., ${ }^{14}$ which use the ICF and the Hypothesis-Oriented Algorithm for Clinicians $(\mathrm{HOAC}-\mathrm{II})^{12}$ to identify a selection of main problems. This allowed goal-setting at all levels of the ICF, according to the needs of the child. The goals were discussed with the child's parents or caretakers, as well as the child's physical therapist. The designs of the programs thereby approached the reality of clinical practice, using a mixture of individual techniques, each targeting a specific problem of the child at that particular moment. The choice of the appropriate techniques to achieve a specific goal was performed according to the evidence-table designed by Franki et al. in previous systematic reviews. ${ }^{2,3}$ Table 1 provides an overview of the similarities and differences between the programs.

Participants were evaluated before and after the period of usual care, as well as after each treatment program using the gross motor function measure, ${ }^{17}$ standardized clinical examination and threedimensional gait analysis.

The Gross Motor Function Measurement- 88 is a standardized clinical instrument to evaluate change 


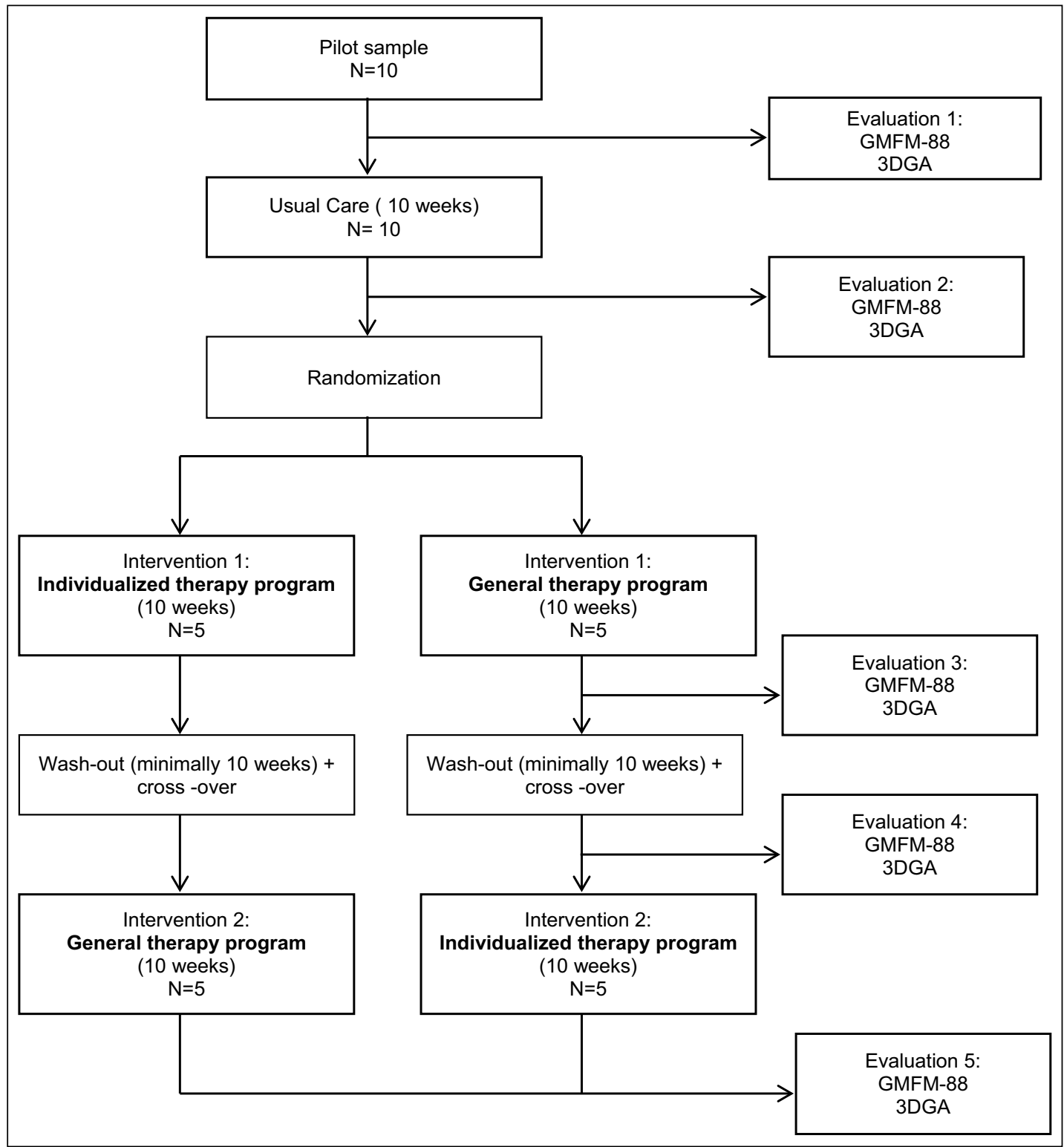

Figure I. Study design.

in gross motor function in children with cerebral palsy. It fulfills the criteria of reliability and validity with respect to responsiveness to change. ${ }^{18-20}$ The gross motor function evaluations were performed by the first author of this study, but videotaped. Two independent assessors, blinded to the set-up of the study, scored the video. The average of both video-scores was used.
Clinical examination and three-dimensional gait analysis were performed according to the standardized protocol used at the Laboratory of Clinical Motion Analysis of the hospital. An independent assessor, blinded to the study, performed both assessments. Clinical examination included measurements of range of motion (goniometrical measurements), spasticity (Modified Ashworth 
Table I. Differences and similarities between the general, aim-oriented program and the individually defined targeted programs.

\begin{tabular}{|c|c|c|}
\hline & General treatment program & Individually defined, targeted treatment programs \\
\hline Differences & $\begin{array}{l}\text { - The same program for all children. } \\
\text { Based on the most common problems of } \\
\text { ambulant children with bilateral spastic } \\
\text { cerebral palsy. } \\
\text { - Aspecific, based on general aims. } \\
\text { - A predefined set of exercises to improve } \\
\text { strength, selectivity and mobility and gross } \\
\text { motor function. } \\
\text { - The results of the three-dimensional gait } \\
\text { analysis were not used to define of the } \\
\text { program. } \\
\text { - The program was not discussed with the } \\
\text { therapist and parents. }\end{array}$ & $\begin{array}{l}\text { - Different for all children. } \\
\text { - Based on the results of the individual } \\
\text { assessments. } \\
\text { - Specific, tailored to the individual problems. } \\
\text { - Goal-oriented. } \\
\text { - Depending on the needs of the child, the } \\
\text { exercises targeted the different levels of the } \\
\text { ICF. } \\
\text { - Based on structured clinical reasoning using } \\
\text { the HOAC-Il and the ICF. } \\
\text { - The results of the three-dimensional gait } \\
\text { analysis were included to define the program. } \\
\text { - The program was discussed with the parents } \\
\text { and the therapist. }\end{array}$ \\
\hline \multirow[t]{4}{*}{ Similarities } & $\begin{array}{l}\text { - Respected the basic principles for } \\
\text { evidence-based physical therapy regarding } \\
\text { intensity and repetitions: }\end{array}$ & $\begin{array}{l}\text { Respected the basic principles for evidence- } \\
\text { based physical therapy regarding intensity and } \\
\text { repetitions: }\end{array}$ \\
\hline & $\begin{array}{l}\text { Stretching: } 3 \text { repetitions of } 30 \text { seconds; } \\
\text { Strength was trained in series of } 3 \\
\text { times } 12 \text { to } 15 \text { repetitions; }\end{array}$ & $\begin{array}{l}\text { Stretching: } 3 \text { repetitions of } 30 \text { seconds; } \\
\text { Strength was trained in series of } 3 \text { times } 12 \\
\text { to } 15 \text { repetitions; }\end{array}$ \\
\hline & $\begin{array}{l}\text { Functional exercises were not } \\
\text { restricted in repetition number. }\end{array}$ & $\begin{array}{l}\text { Functional exercises were not restricted in } \\
\text { repetition number. }\end{array}$ \\
\hline & $\begin{array}{l}\text { - Executed by the child's private physical } \\
\text { therapist, familiar to the child. } \\
\text { - At the child's usual frequency and duration } \\
\text { of therapy. }\end{array}$ & $\begin{array}{l}\text { - Executed by the child's private physical } \\
\text { therapist, familiar to the child. } \\
\text { - At the child's usual frequency and duration of } \\
\text { therapy. }\end{array}$ \\
\hline
\end{tabular}

ICF: International Classification of Functioning, Disability and Health; HOAC: Hypothesis-Oriented Algorithm for Clinicians.

Scale and Tardieu), selectivity (selective motor scale) and muscle strength (manual muscle testing). ${ }^{21-23}$ For gait analysis, information on kinematics, kinetics and spatial-temporal data was collected by a 16-camera VICON system (Nexus PluginGait marker set, Oxford Metrics, Oxford, UK), two ATMI forceplates (Advanced Medical Technology, Inc, Watertown, MA, USA) and a 16-channel electromyograpy (EMG) device (ZeroWire, Cometa, Milano, Italy). Three representative trials were selected. Specific gait parameters were automatically extracted using a custom-made Matlab graphical user interface (Mathworks ${ }^{\circledR}$, Natick, MA, USA). The results of the children were compared with the means and standard deviations of a reference group. Our reference data was based on the data obtained from a control group of 55 typically developing children, with a mean age of 10.94 years, range between the ages of four years and 18 years.

Outcome parameters were derived at two different levels (Figure 2).

At a first, general level, overall therapy success was evaluated using outcome parameters that provide a general outcome description. From the Gross Motor Function Measure, the total and dimensional percentage scores were extracted. Regarding gait analysis, time and distance parameters, the Movement Analysis Profiles and the Gait Profile Score were calculated. ${ }^{24}$ The average scores from the left and right sides were used. The Movement Analysis Profiles were calculated as the root mean square error between the point-by-point comparison of the lower limb joint angle and the averaged joint angle of the reference group. Calculation of the average of all lower limb joint 


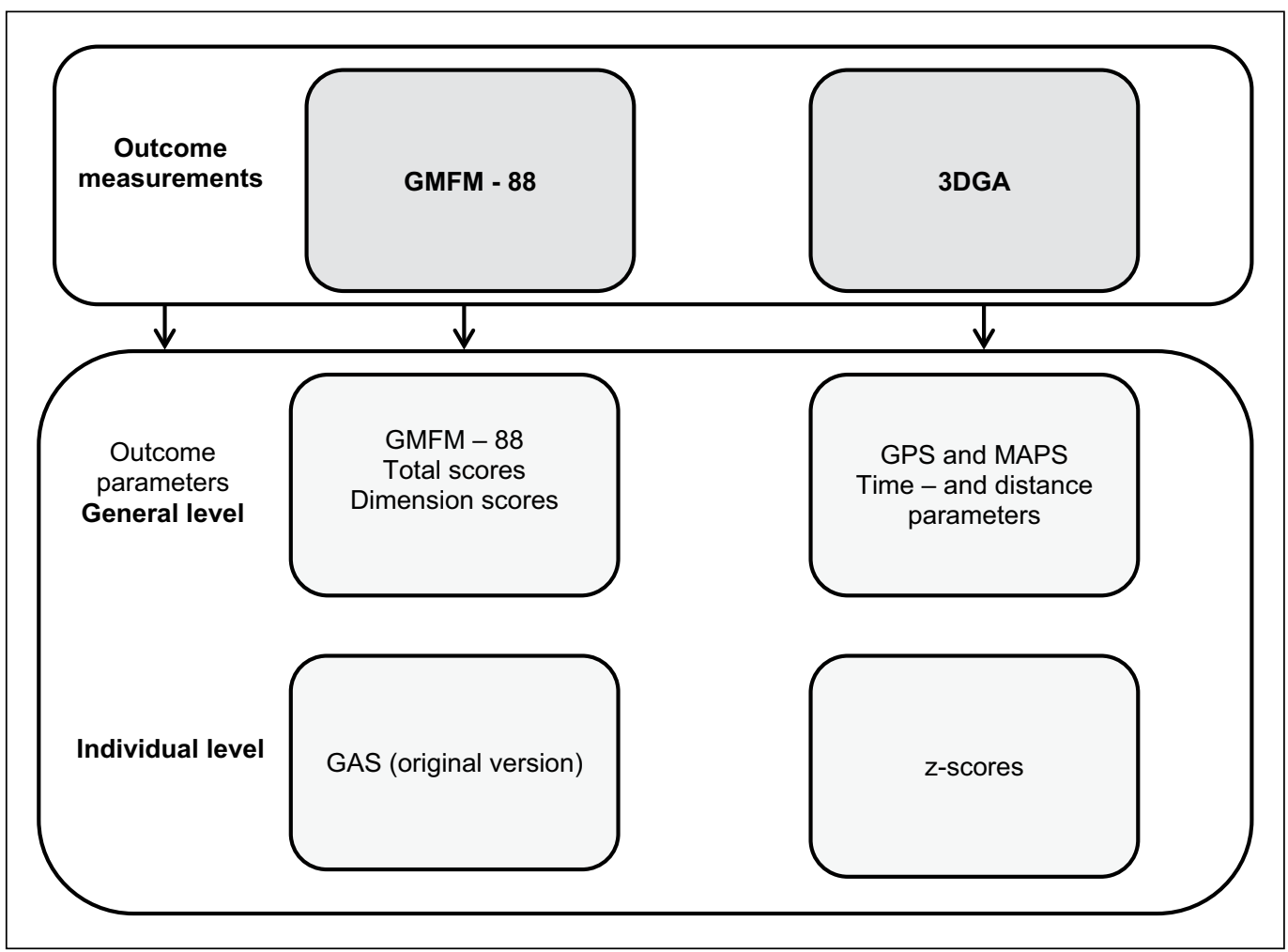

Figure 2. Outcome measurements and parameters.

3DGA: Three-Dimensional Gait Analysis; GAS: Goal Attainment Scale; GMFM-88: Gross Motor Function Measurement; GPS: Gait Profile Score; MAPS: Movement Assessment Profiles.

angles results in the Gait Profile Score, which summarizes the overall severity of gait pathology. ${ }^{24}$

Second, individual goal achievement was evaluated before and after the programs.

For the individual, functional goals extracted from the Gross Motor Function Measure-88, the original version of the Goal Attainment Scale (GAS) was used, as developed by Kirusek et al. ${ }^{25}$ and used in several pediatric rehabilitation studies. ${ }^{26,27}$ Before the start of the programs, individual goals based on specific goal items of the Gross Motor Function Measure-88, were formulated according to the specific, measurable, achievable, realistic/ relevant and timed (SMART)-principle. ${ }^{28}$ A score between -2 and 2 was given depending on the goal achievement. Subsequently, a converted T-score and the percentage of achieved goals $(\geqslant 0)$ were calculated.
Individual goal achievement of the specific goals derived from gait analysis was evaluated using z-scores and proportional changes. As a first step, two to eight specific deviated gait parameters were identified as specific goals for each child. Each goal was represented by a relevant data point that was expected to improve, such as maxima, minima, range of motion and angles at specific events in the gait cycle. These data points were defined based on literature and by clinical experts in gait analysis of children with cerebral palsy. ${ }^{29,30}$ The parameters were converted into z-scores. A z-score represents the deviation from a reference group $(\mathrm{z}=$ (mean child - mean typical child)/standard deviation (SD) typical child). To evaluate the z-scores, the absolute difference to zero, in either direction, was considered as an equal deviation from typical gait. Following standard clinical practice, we 
assumed that a deviation greater than 1 could be considered as pathological. Therefore, all z-scores smaller than 1 were eliminated. ${ }^{29}$ Consequently, the median z-scores of the specific goals were calculated. Z-scores are non-dimensional and, therefore, allow the comparison of different parameters. Finally, the proportional change was calculated (proportional change $=(\mid$ pre median $\mathrm{z}$-score $\mid-$ |postmedian z-score| / |premedian z-score|)). This percentage score allowed a description of therapy success. A positive proportional change described a successful therapy and a negative proportional change indicated an unsuccessful therapy. For the individually defined programs, these individual goals were taken into account to design the programs. For the general programs, the individual goals were only considered as outcome parameters, but not specifically taken into account to during the programs.

Statistical analyses were performed in different steps. First, other treatment modalities, including the use of orthosis, aquatic and horse-riding therapy, during the different intervention periods were compared using a McNemar test. Similarly, differences in the frequency and duration of the physical therapy sessions were tested using the relatedsamples Wilcoxon Signed-Rank test. Secondly, the median and interquartile range (IQR) of all parameters before and after the programs were calculated as appropriate. A related-samples Wilcoxon signed rank analysis was applied to calculate within-group differences of pre- and postvalues. In order to evaluate the effect between the different interventions, a related-samples Friedman's two-way analysis of variance by rank analysis with post-hoc relatedsamples Wilcoxon signed rank analysis was applied. This analysis compared the difference scores of the outcome parameters (difference score $=$ Pre - Post), further described as between-program differences.

All statistics were performed in SPSS 21 IBM SPSS Statistics for Windows, Version 21.0. (IBM Corp, Armonk, NY, USA).

\section{Results}

Patient characteristics, as well as duration, frequency of therapy and the use of orthosis are reported in Table 2 and were not significantly different between the different periods.

The results of the diaries provided during the period of usual care show that $55.8 \%$ of therapy time was dedicated at impairment level. Thereby, $22.5 \%$ (interquartile range (IQR) 16.7-28.6) targeted joint mobility and muscle length and $33.3 \%$ (IQR 23.9-35.9) strength. A median of 48.1\% (IQR $35.6-54.8 \%$ ) of therapy time was used to train activities. Only $1.55 \%$ (IQR $0-6.5 \%$ ) of therapy time addressed specific participation restrictions.

The median total percentage scores of the Gross Motor Function Measure-88 showed only very limited and no significant changes, but a slight increase after the individualized program, from 93.5 to $95.5 \%$ (Table 3 ).

Dimension D and E showed a slight decrease during the period of usual care, but an increase after the period of individualized therapy. Evaluating the between-program differences revealed no significance.

Considering time and distance parameters showed significant improvements on step and stride length ( $p=0.022$ and 0.017 , respectively) after the period of individualized therapy. Betweenprogram effects were, therefore, also significant for step length in the advantage of the individualized therapy program $(p=0.045)$. Changes in Gait Profile Score and Movement Assessment Profile Scores are provided in Table 4. Significant changes were found after the individualized program, for the pelvis at the transversal plane $(p=0.047)$, while for the general program differences were found for the pelvis in the coronal plane $(p=0.047)$. Betweenprogram changes were only found for the knee in the sagittal plane and this in the advantage of the individualized therapy program $(p=0.047)$. The results of the Gait Profile Score showed slight progress after the individualized program, whereas deterioration was registered after the general program. No changes were measured after the period of usual care.

Functional goal attainment was higher after the individualized than after the general therapy program: 48.0 (IQR 43.3-59.3) compared with 43.5 (IQR 30.3-43.5) ( $p=0.285)$. Additionally, the averaged percentage of achieved goals was 50.0 
Table 2. Patient characteristics.

\begin{tabular}{|c|c|c|c|}
\hline \multicolumn{4}{|l|}{ Patient characteristics } \\
\hline Age (median + IQR) & \multicolumn{3}{|c|}{6.2 years $(4.6-7.0$ years $)$} \\
\hline Gender ( $n$ ) (male/female) & \multicolumn{3}{|c|}{$6 / 4$} \\
\hline GMFCS $(n)(I / I I)$ & \multicolumn{3}{|l|}{$5 / 5$} \\
\hline \multirow[t]{2}{*}{ History of BTX-A injections ( $n$ ) } & \multicolumn{3}{|l|}{4} \\
\hline & UC & GT & IT \\
\hline \multicolumn{4}{|l|}{ Use of day orthosis (n) } \\
\hline$>50 \% /$ day & 8 & 7 & 7 \\
\hline$<50 \% /$ day & 0 & 2 & 2 \\
\hline no & 2 & I & 1 \\
\hline \multicolumn{4}{|l|}{ Use of night orthosis (n) } \\
\hline$>50 \% /$ night & I & 2 & 0 \\
\hline$<50 \% /$ night & 0 & 2 & 4 \\
\hline no & 9 & 6 & 6 \\
\hline Frequency of PT (times/week) Med (IQR) & $2(2-3)$ & $3(2-3)$ & $3(2-4)$ \\
\hline Duration of PT (minutes/session) Med (IQR) & $40(30-60)$ & $40(30-60)$ & $40(30-60)$ \\
\hline Total PT (minutes/week) & $105(80-120)$ & $120(90-128)$ & $120(90-123)$ \\
\hline
\end{tabular}

BTX-A: Botulinum Toxin A; GMFCS: Gross Motor Function Classification System; GT: period of general treatment; IQR: interquartile range; IT: period of individualized, targeted treatment; PT: physical therapy; UC: period of usual care.

(IQR 25.0-100) after the individualized program, compared with 41.7 (IQR 0-75) for the general program $(p=0.589)$. Nevertheless, none of the programs caused treatment success (GAS $\geqslant 50$ ) and the differences were not statistically significant. Table 5 provides an overview of the changes in z-scores for the goals derived from the gait analysis after both training periods. This analysis revealed a median difference in z-score (pre-post) of 0.279 and 0.419 after the period of general and individualized therapy program, respectively. This was translated in a proportional change of $12 \%$ and $19 \%$, respectively. These changes, however, were not significantly different.

\section{Discussion}

The effects of the present study demonstrated a tendency in favor of the individually defined, targeted program on gait and gross motor function. On a general level, significant advantageous results were found for the individualized program on step length and for the movement assessment profiles of the knee in the sagittal plane. At an individual level, goal achievement was non-significantly higher after the individualized program compared with the general program, and this for both the functional goals as the goals based on gait analysis.

The applied approach was representative for the reality of clinical practice, which usually makes use of a mixture of individual techniques, each targeting a specific problem. It differed from more recent goal-oriented, activity-focused interventions that usually apply a 'top-down' approach within the IFC. ${ }^{31}$ The clinical reasoning strategy used to define the individually tailored programs was based on a horizontal approach and, therefore, considered all dimensions of the model of equal importance. It aimed to specifically address the thoughts behind the dynamic and interactive concept of the ICF and, thereby, also the multidimensional difficulties following cerebral palsy. Gait rehabilitation was an essential part of our programs and a large part of our outcome parameters consisted of gait measures. This was motivated by previous research that highlighted that both gait speed and the amount of gait pathology adversely affected quality of life, as reported by the child and their proxy. ${ }^{32}$ This unique but realistic concept can be considered as a major strength of our study. 


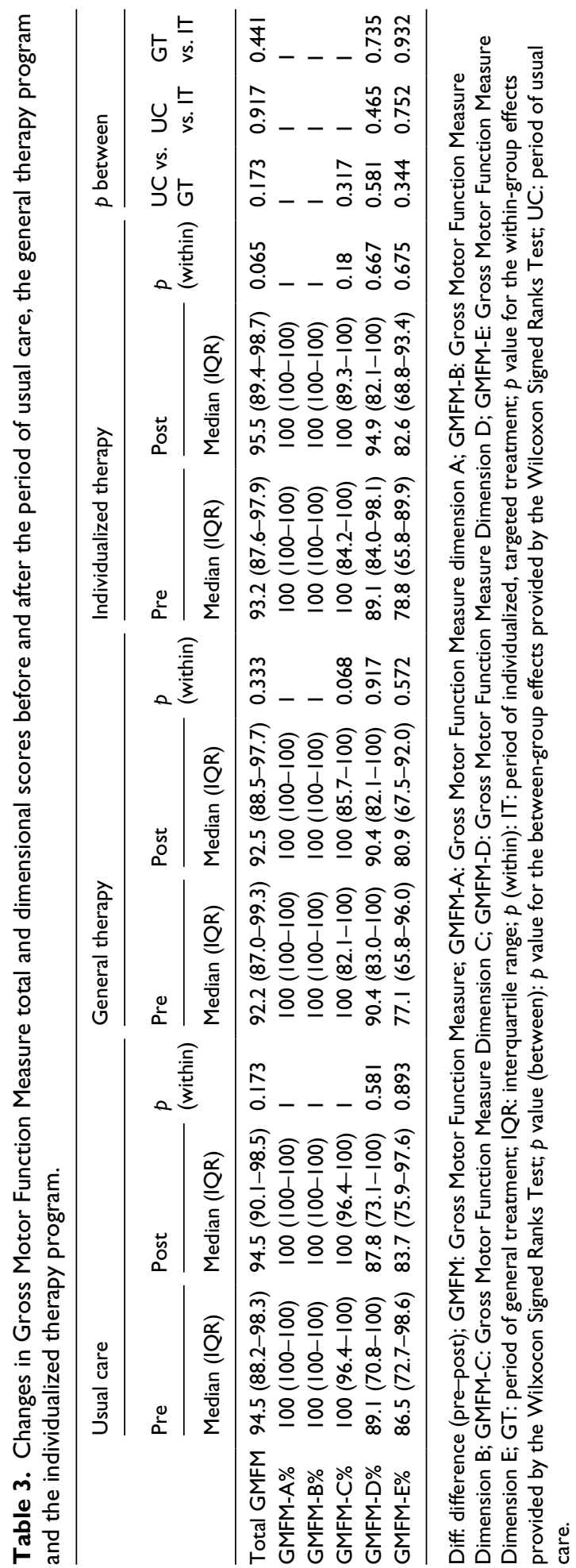




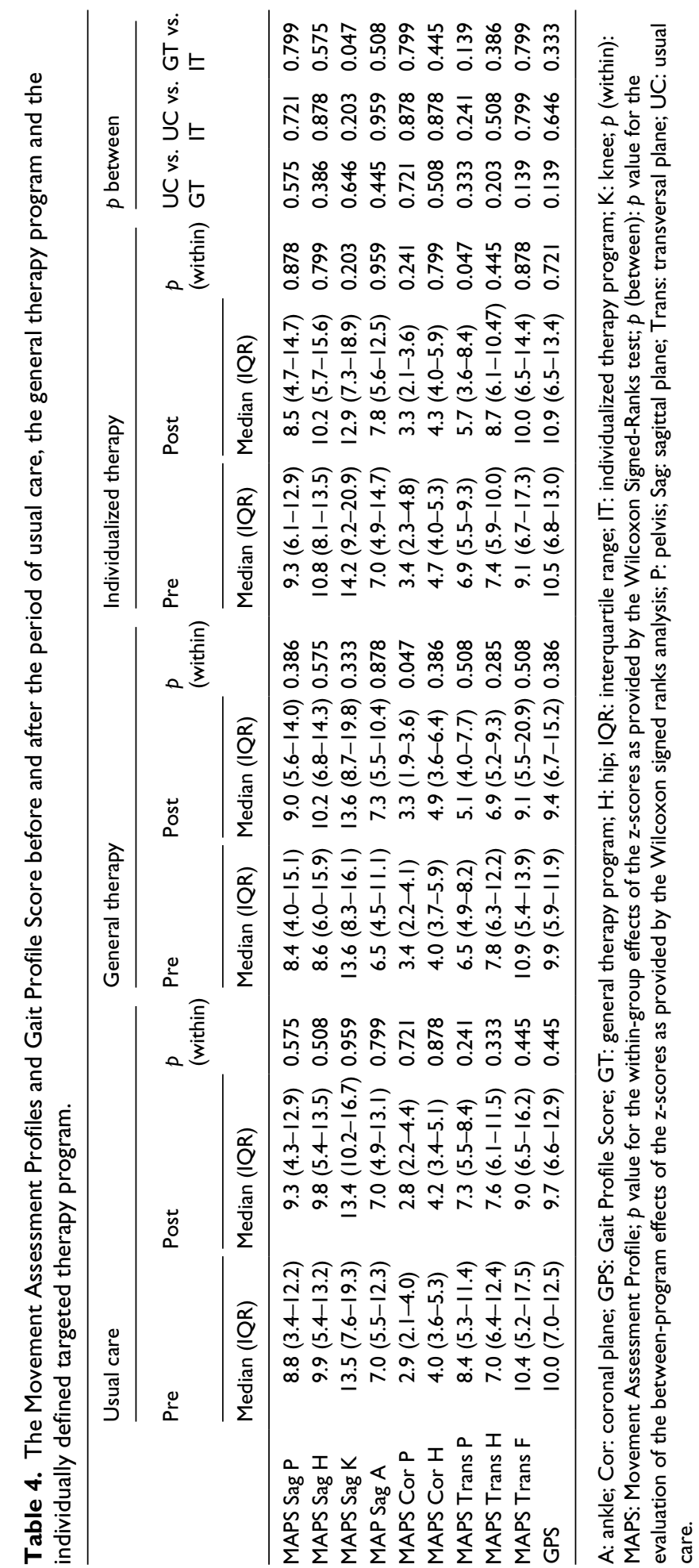


Table 5. Overview of the changes in z-scores and the converted proportional changes as derived from the individual goals based on the results of the three-dimension gait analysis.

\begin{tabular}{|c|c|c|c|c|c|c|c|c|c|c|c|}
\hline \multirow[t]{2}{*}{ Child } & \multicolumn{5}{|c|}{ General program } & \multicolumn{5}{|c|}{ Individualized program } & \multirow[t]{2}{*}{$p$ (between) } \\
\hline & Pre & Post & Diff & PC & $p$ (within) & Pre & Post & Diff & PC & $p$ (within) & \\
\hline 1 & 2.967 & 3.142 & -0.175 & $-6 \%$ & 0.368 & 2.718 & 2.656 & 0.062 & $2 \%$ & 0.169 & 0.333 \\
\hline 2 & 1.646 & 1.184 & 0.462 & $28 \%$ & & 1.669 & 0.738 & 0.931 & $56 \%$ & & \\
\hline 3 & 2.334 & 1.576 & 0.759 & $33 \%$ & & 2.271 & 1.629 & 0.641 & $28 \%$ & & \\
\hline 4 & 3.093 & 2.475 & 0.618 & $20 \%$ & & 2.410 & 1.672 & 0.738 & $31 \%$ & & \\
\hline 5 & 1.592 & 2.026 & -0.434 & $-27 \%$ & & 2.449 & 2.536 & -0.088 & $-4 \%$ & & \\
\hline 6 & 1.439 & 0.747 & 0.693 & $48 \%$ & & 3.349 & 3.003 & 0.347 & $10 \%$ & & \\
\hline 7 & 1.485 & $0.74 I$ & 0.744 & $50 \%$ & & 1.867 & 1.179 & 0.688 & $37 \%$ & & \\
\hline 8 & 1.472 & 1.886 & -0.414 & $-28 \%$ & & 1.453 & 0.961 & 0.492 & $34 \%$ & & \\
\hline 9 & 2.134 & 2.038 & 0.096 & $4 \%$ & & 2.703 & 2.804 & -0.101 & $-4 \%$ & & \\
\hline 10 & 3.263 & 4.127 & -0.864 & $-26 \%$ & & 2.788 & 3.681 & -0.893 & $-32 \%$ & & \\
\hline 50th (median) & 1.889 & 1.817 & 0.278 & $12 \%$ & & 2.429 & 2.104 & 0.419 & $19 \%$ & & \\
\hline 25 th & 1.481 & 1.070 & -0.419 & $-27 \%$ & & 1.817 & 1.124 & -0.090 & $-4 \%$ & & \\
\hline 75th & 2.990 & $2.64 I$ & 0.705 & $36 \%$ & & 2.735 & 2.853 & 0.700 & $35 \%$ & & \\
\hline
\end{tabular}

Diff: difference between the pre- and postvalue; $p$ (within): $p$ value for the within-group effects of the z-scores as provided by the Wilcoxon signed ranks test; $p$ (between): $p$ value for the evaluation of the between-program effects of the z-scores as provided by the Wilcoxon signed ranks analysis; PC: proportional change, calculated by (z pre $-z$ post)/z pre.

Another strength of the study was the randomized cross-over design, allowing comparison of individual changes. The length of the wash-out period, thereby, was the same as the duration of the program. Wash-out periods are required assuming that therapy effects are still present for a certain period after the intervention. In our recent systematic literature review, none of the studies using follow-up periods detected treatment effects lasting longer than the duration of the intervention itself. $^{2}$ Additionally, in the current study, only the contents of the programs were adjusted and the researchers did not interfere with the frequency and duration of the programs. These considerations allowed us to be confident that a 10 weeks washout period is sufficient to avoid overflow effects.

Nevertheless, only a limited number of parameters showed statistical significant changes. A first and major reason might be the small sample size. Although the set-up of the study was controlled and randomized, the sample size of 10 children provided low power. Post-hoc power calculation using SAS Power and Sample Size 3.1 (SAS institute Inc, Cary, NC, USA) revealed the gait profile score as being most vulnerable for the limited power. Considering a change score of $3^{\circ}$ on the gait profile score as clinically relevant, provided $8 \%$ power. To be able to detect this change, future studies should use between 72 ( $80 \%$ power) to 91 participants $(90 \%)$. The Gross Motor Function Measure- 88 was identified as least vulnerable for limited power. When using the Gross Motor Function Measure- 88 as an outcome measure, future studies should use between 12 (80\% power) to 15 participants $(90 \%)$ to detect a change of $3 \%$.

A second restriction might be related to the fact that both programs were executed by the child's personal physical therapist. Besides the strict follow-up, influencing factors as cooperation of the therapist and correct execution of the programs still existed and were probably important confounding variables. The adequate follow-up could probably not completely control the correct appliance and execution of the prescribed programs. During the period of usual care, regular physical therapy sessions were continued. Therefore, the contents of the programs were dependent of the physical therapist responsible for the child and a 
large variation in therapy contents was possible. A diary was used to be able to monitor therapy contents without interfering with the quality and contents of therapy. Follow-up visits might have influenced therapy behavior and, therefore, diaries were preferred. Although the diary was a useful and objective tool, the physical therapists reported that filling the diary was time-consuming and caused a relatively large administrative burden. Therefore, diaries were avoided during the periods of interventions. The need for a diary during the periods of intervention was not strong as the programs were predefined using specific exercises. Therefore, follow-up during the interventions was performed using regular visits and phone-calls. Nevertheless, although the therapy programs were developed with more than $50 \%$ of the exercises targeting activity level, this did not necessary mean that therapy time was divided accordingly. Providing a diary during the periods of interventions might have provided more precise insight into the follow-up of the programs.

A third limitation was the duration of the applied interventions. Ten weeks was a relatively short period to reach significant improvements. Guidelines by the National Strength and Conditioning Association stated that strength training should be applied 2-4 times a week on non-consecutive days for at least 8-20 weeks in order to find an increase in muscle strength. ${ }^{33}$ Therefore, a study of longer duration could have resulted in greater benefits. Additionally, longer-term studies allow an evaluation at participation level. ${ }^{34}$ As the study period of 10 weeks was too short to expect significant changes at participation level, outcome evaluation did not include participation measures. ${ }^{34}$ Nonetheless, the design of the individualized integrated discussions with parents, children and therapists. In that way, individual participation limitations were considered during the development of the programs and were considered of equal value among the assessment measures when designing the programs.

Additionally, while the inclusion criteria allowed inclusion of children with Gross Motor Function Classification Level III, no children functioning at this level could be included for this intervention study. Children with Gross Motor
Functional Classification Level III are more frequently in need of tone-reduction by means of botulinum toxin and, therefore, we were not able to include these children during the predefined window of six months post-botulinum toxin.

The results demonstrated very limited difference but advantageous results of the interventions compared with the period of usual care. This might be explained by the variation in therapy as reported by the different therapists. Remarkably however, as all children were ambulant and thus very functional, the percentage of time spent on impairment level (range between $40.3 \%$ and $70.3 \%$ of therapy time) was relatively high during the period of usual care. During the periods of intervention, the number of exercises targeting activity level was usually higher than 50\%. Although this implies that during the interventions the average therapy time spent at activity level was probably higher than $50 \%$, it might be a future recommendation to monitor therapy time more accurately during the periods of intervention as well.

The proportional change scores allowed us to describe therapy success by explaining the gain or loss in performance. However, one needs to be careful with the interpretation of a proportional change score because it is easily influenced by extraneous factors (such as the standard deviation of the control group), and it may therefore overestimate outcome results.

The study results of the present study are concurrent with the previous experience of our research group, in which we evaluated the effectiveness of an individually defined approach. ${ }^{9}$ This study showed fundamental differences with the present study: the order of the programs was not randomized and they were of shorter duration ( 6 weeks). Additionally, the present structured clinical reasoning was not yet the basis for the individual programs.

The recent review by Brogren et al. concluded that the scientific evidence regarding goal-setting is inconclusive. ${ }^{10}$ Also, Law et al. could not find significant differences between a context-focused therapy containing goal-setting, and child and activity focused therapy without goal-setting. ${ }^{35}$ However, these studies used goal-setting within an activity-focused approach only. 
This again supports our hypothesis that a successful treatment approach is probably multifactorial and depends on several aspects like correct appliance of techniques, adequate goal and priority setting, frequency and duration of therapy, timely tone-reduction, attention for posture and positioning and maybe many others. Future research studies should probably continue to look for the effectiveness of individually tailored approaches addressing the heterogeneous nature of cerebral palsy, not only in individual goal-setting, but also in the choice of techniques and approaches.

In conclusion, the results can only provide an indication of the additional value of goal-setting within an integrative approach. To confirm our hypothesis however, future studies should include sample sizes of minimally 72 participants, evaluate long-term effects and use individual goal-achievement measures.

\section{Clinical messages}

- In children with cerebral palsy, improvements in gait and gross motor function showed a non-significant tendency to be greater after the use of an individualized rather than a general treatment program.

- A sample size of between 72 and 91 participants is needed to confirm this tendency, providing $80 \%$ and $90 \%$ power, respectively.

\section{Acknowledgements}

The authors would like to thank Sophie Van Hoey and Sofie Chevalier for the blinded scoring of the GMFM- 88 video measurements and Dorian Conincx for her valid contribution to the data-analysis. We thank Dr Ellen Deschepper of the Department of Public Health, Biostatistics Unit of Ghent University, for her professional advice regarding the data-analysis.

\section{Conflict of interest}

The authors confirm that all authors and persons in the acknowledgments have seen and approved the manuscript. Each author has participated sufficiently in the work to take public responsibility for the content. The authors state that there are no conflicts of interests and funding.

\section{Funding}

This research received no specific grant from any funding agency in the public, commercial, or not-for-profit sectors.

\section{References}

1. Anttila H, Suoranta J, Malmivaara A, Mäkelä $M$ and Autti-Rämö I. Effectiveness of physiotherapy and conductive education interventions in children with cerebral palsy. Am J Phys Med Rehab 2008; 87(6): 478-501.

2. Franki I, Desloovere K, De Cat J, et al. The evidencebase for basic physical therapy techniques targeting lower limb function in children with cerebral palsy: a systematic review using the International Classification of Functioning, Disability and Health as a conceptual framework. J Rehabil Med 2012; 44(5): 385-395.

3. Franki I, Desloovere K, De Cat J, et al. The evidencebase for conceptual approaches and additional therapies targeting lower limb function in children with cerebral palsy: a systematic review using the ICF as a framework. $J$ Rehabil Med 2012; 44(5): 396-405.

4. Ketelaar M, Vermeer A, Hart H, Van Petegem-Van Beek $\mathrm{E}$ and Helders PJ. Effects of a functional therapy program on motor abilities of children with cerebral palsy. Phys Ther 2001; 81(9): 1534-1545.

5. Bower E, McLellan DL, Arney J and Campbell MJ. A randomised controlled trial of different intensities of physiotherapy and collaborative goal-setting in children with cerebral palsy. Dev Med Child Neurol 1996; 38: 226-237.

6. Bower E, Michell D, Burnett M, Campbell MJ and McLellan DL. Randomized controlled trial of physiotherapy in 56 children with cerebral palsy followed for 18 months. Dev Med Child Neurol 2001; 43(1): 4-15.

7. Löwing K, Bexelius A and Carlberg EB. Goal-directed functional therapy: A longitudinal study on gross motor function in children with cerebral palsy. Disabil Rehabil 2010; 32(11): 908-916.

8. Löwing K, Bexelius A and Brogren Carlberg E. Activity focused and goal directed therapy for children with cerebral palsy - Do goals make a difference? Disabil Rehabil 2009; 31(22): 1808-1816.

9. Van den Broeck C, De Cat J, Molenaers G, et al. The effect of individually defined physiotherapy in children with cerebral palsy (CP). Eur J Paediatr Neurol 2010; 14(6): 519-525.

10. Brogren Carlberg E and Löwing K. Does goal setting in activity-focused interventions for children with cerebral palsy influence treatment outcome? Developmental Med Child Neurol 2013; 55 (Suppl 4): 47-54.

11. Levack WMM, Dean SG, Siegert RJ and McPherson KM. Purposes and mechanisms of goal planning in rehabilitation: 
The need for a critical distinction. Disabil Rehabil 2006; 28(12): 741-749.

12. Rothstein JM, Echternach JL and Riddle DL. The hypothesis-oriented algorithm for Clinicians II: A guide to patient management. Phys Ther 2003; 83(5): 455-470.

13. World Health Organization. Nederlandse vertaling $I C F$ $C \& Y$ version. $2008,1 \mathrm{p}$.

14. Franki I, De Cat J, Deschepper E, et al. A clinical decision framework for the identification of main problems and treatment goals for ambulant children with bilateral spastic cerebral palsy. Res Dev Disabil 2014; 35(5): 1160-1176.

15. Altman DG and Bland JM. Statistics notes - Treatment allocation by minimisation. BMJ 2005; 5: 843 .

16. O’Neil ME, Fragala-Pinkham MA, Westcott SL, et al. Physical therapy clinical management recommendations for children with cerebral palsy - spastic diplegia: achieving functional mobility outcomes. Ped Phys Ther 2006; 18(1): 49-72.

17. Russell DJ, Rosenbaum PL and Gowland C. Gross Motor Function Measure manual. Ontario, Canada: Wiley Blackwell Publishing, 1993.

18. Bjornson KF, Graubert CS, Buford V and McLaughlin J. Validity of the Gross Motor Function Measure. Ped Phys Ther 1998; 10: 43-47.

19. Russell DJ, Rosenbaum PL, Cadman DT, Gowland C, Hardy S and Jarvis S. The Gross Motor Function Measure: A means to evaluate the effects of physical therapy. Dev Med Child Neurol 1989; 31: 341-352.

20. Russell DJ, Avery LM, Rosenbaum PL, Raina PS, Walter $\mathrm{SD}$ and Palisano RD. Improved scaling of the Gross Motor Function Measure for children with cerebral palsy: Evidence of reliability and validity. Phys Ther 2000; 8: 873-885.

21. Fosang AL, Galea MP, McCoy AT, Reddihough DS and Story I. Measures of muscle and joint performance in the lower limb of children with cerebral palsy. Dev Med Child Neurol 2003; 45(10): 664-670.

22. Damiano DL, Quinlivan JM, Owen BF, Payne P, Nelson $\mathrm{KC}$ and Abel MF. What does the Ashworth scale really measure and are instrumented measures more valid and precise? Dev Med Child Neurol 2002; 44: 112-118.

23. Scholtes Vanessa AB, Becher JG, Beelen A and Lankhorst GJ. Clinical assessment of spasticity in children with cerebral palsy: a critical review of available instruments. Dev Med Child Neurol 2006; 48: 64-73.
24. Baker R, McGinley JL, Schwartz MH, et al. The Gait Profile Score and Movement Analysis Profile. Gait \& Posture 2009; 30(3): 265-269.

25. Kiresuk TJ, Smith A and Condillo JE. Goal attainment scaling: Applications, theory and measurement. New Jersey: Psychology Press (Taylor \& Francis Group) 1994.

26. Palisano RJ. Validity of goal attainment scaling in infants with motor delays. Phys Ther 1993; 73: 651-658.

27. Mailloux Z, May-Benson TA, Summers CA, et al. Goal attainment scaling as a measure of meaningful outcomes for children with sensory integration disorders. Am J Occupat Ther 2007; 61 (2): 254-259.

28. Bovend'Eerdt TJ, Botell RE and Wade DT. Writing SMART rehabilitation goals and achieving goal attainment scaling: a practical guide. Clin Rehabil 2009; 23(4): 352-361.

29. Gage JR, Schwartz MH, Koop SE and Novacheck TF. The identification and treatment of gait problems in cerebral palsy. London: Mc Keith Press, 2009.

30. Winter DA. Biomechanics and motor control of human movement. Hoboken, NJ, USA: John Wiley \& Sons, Inc., 2009.

31. Novak I, McIntyre S, Morgan C, et al. A systematic review of interventions for children with cerebral palsy: state of the evidence. Dev Med Child Neurol 2013; 55(10): 885-910.

32. Jaspers E, Verhaegen A, Geens F, Van Campenhout A, Desloovere K and Molenaers G. Lower limb functioning and its impact on quality of life in ambulatory children with cerebral palsy. Eur J Paed Neurol 2013; 17(6): 561-567.

33. Faigenbaum AD, Kraemer WJ, Blimkie CJR, et al. Youth resistance training: Updated position statement article from the National Strength and Conditioning Association. J Strength Condit Res 2009; 23: 60-79.

34. King GA, Law M, King S, et al. Measuring children's participation in recreation and leisure activities: construct validation of the CAPE and PAC. Child Care Health Dev 2007; 33(1): 28-39.

35. Law M, Darrah J, Pollock N, et al. Focus on function: A cluster, randomized controlled trial comparing childversus context-focused intervention for young children with cerebral palsy. Dev Med Child Neurol 2011; 53: 621-629. 
Reproduced with permission of the copyright owner. Further reproduction prohibited without permission. 